was also to die in the Gallipoli campaign. For Moseley himself wrote to Rutherford "the French point of view [of physics] is essentially different from the English. Where we try to find models or analogies, they are content with laws." But irrespective of whatever truth there may have been in this comment, physicists of all nationalities who are interested in the development of their subject owe Professor Heilbron a debt for his excellent biography. And if this review seems to have been coloured by Moseley's own opinion that "reviews in Nature are usually such pure honey", in this case both the subject and his biographer deserve it.

\section{Evolution of populations}

Genetical Structure of Populations. By Kenneth Mather. Pp. viii + 197. (Chapman and Hall: London, 1973.) £4.00.

Professor MAther's latest book is a survey of topics in population genetics, intended for advanced undergraduate and postgraduate students seeking a general orientation. The subject is the way in which proportions of genetic types change from generation to generation in response to various factors, and the effect of these changes on the status of the population in relation to its environment. The approach is quantitative but not mathematical, being directed to readers with biological education and interests. The mathematical level varies considerably from chapter to chapter with more extensive algebraic treatment of traditional topics such as the Hardy-Weinberg law and its generalisations.

After a short introduction the style of the work is established in the second chapter which reviews the causes of viariability in biology. Professor Mather assumes that readers understand the basic facts of inheritance, and devotes himself to a detailed exposition of the way in which these affect population changes. Although the results of algebraic analysis are stated, most proofs are omitted. Later in the book, chapter 6 on "Life Cycles and Genetic Systems" is a mine of interesting information but contains no mathematical analysis at all. Chapter 7 on "Genic Balance and Genetic Architecture" involves quantitative statements about the population effects of the manner in which the genetic system acts on numerical characteristics, but again contains no proofs.

Chapters 3 and 4 ("Equilibria" and "Theory of Variability") reveal the strength and weakness of the attempt to discuss a quantitative subject while eschewing advanced mathematics. In mathematical books on population genetics (among which Moran's Statistical Processes of Evolutionary Theory,

\section{Reproduction of marine invertebrates \\ by R. P. Dales}

Reproduction of Marine Invertebrates. Vol. 1: Acoelomate and Pseudocoelomate Metazons. Edited by Arthur C. Giese and John S. Pearse. Pp. xi+546. (Academic: New York and London, 1974.) \$38; $£ 13.45$.

THIs useful and scholary work is the first of seven volumes. The first six will give a systematic treatment with a chapter devoted to each class or phylum; the final volume will be devoted to general aspects of reproductive physiology. This first volume is especially useful for its coverage of so many minor but interesting phyla such as Kinorhynchs and Gnathostomulids. But all of the chapters are useful: I found the amount of descriptive detail to have been well judged and the accounts of reproduction and embryonic development to be concise and informative. The appearance of the series is timely for there has been much recent work on reproduction in invertebrate phyla.

References are given in full at the end of each chapter and the volume as a whole is provided with separate indices to authors, subjects and taxonomic names. The volume, containing many line figures and half-tones, is well produced. No zoological library can afford to be without this series.

published in 1962 remains unsurpassed despite many subsequent competitors) the problem of selection, random tendency to fixation, mutation, non-random mating and so on, are treated as separate topics. In nature they are all acting simultaneously, one reinforcing or counteracting the effect of another. Professor Mather makes this clear by detailed examples in a full and well balanced discussion. This chapter leaves the reader with a profound respect for the author's deep knowledge of what is really going on in a natural population and a realisation of how much can be lost through oversimplification.

Two points of substance are worthy of note. It is clear towards the end that Mather believes that competition is always frequency dependent, and it must follow that much recent work in mathematical genetics is of little value since it assumes fixed selection coefficients. He devotes several pages to a discussion of the concept of 'genetic load' which has been so hotly debated in recent years. Mathematicians with an interest in genetics, who have been worried by their inability to see the point of all this, will be pleased that such a distinguished geneticist concludes "it is far from clear that the concept, as it has been defined and used, has much significance at all for the understanding of populations and their structure".

In discussing the theory of variability, Professor Mather applies a statistical analysis of variance to the variation in a numerical trait in a population. He supposes, however, that it contains equal proportions of the alleles at one locus or at each of two loci, and obtains components of free, potential and utilised variability which can be transformed into one another as a result of various effects and which are "acted on" by the factors of selection. These ideas clearly give insight into the heart of Mendelism, the fact that variability provides the basis for evolution. They would explain it completely if, when the gene proportions are not equal, the transformation of components behaves qualitatively in the same way. Now in the case of constant selection depending on two or more loci this is not true. There may be several stable equilibria, each with its domain of attraction, and it is unlikely that the same analysis of the responses of components of variability to selective factors will hold in the neighbourhood of them all. The analysis is therefore too restricted in its validity to be convincing. The weaknes of this chapter and the previous one is the readiness with which it is assumed that the qualitative results of algebra applied to simple systems can be extended to complex systems for which results have not been obtained.

This has led to wrong conclusions in the past, and the student should be warned against the practice rather than led into it. It may well be that when it becomes possible to analyse fully realistic models, the results will prove to be on the lines described by Professor Mather on the basis of his wide knowledge, since factors neglected in (say) the multilocus constant selection model may then appear and eliminate superfluous stable equilibria. It is also probable that a satisfactory mathematical analysis of these problems cannot be achieved through classical algebra and will require the application to genetics of topological considerations such as those introduced by Thom in biology.

This is an admirable textbook for students, containing a masterly exposition of what is really important in our current knowledge of population genetics. In order to go beyond it a student will need to study the mathematical side of the subject at a much higher level, and should be encouraged to regard at least some of the assertions as tentative until they have been proved. Philip Holgate 